\title{
Non-cyanobacterial diazotrophs mediate dinitrogen fixation in biological soil crusts during early crust formation
}

\author{
Charles Pepe-Ranney ${ }^{1}$, Chantal Koechli ${ }^{2}$, Ruth Potrafka ${ }^{3}$, Cheryl Andam², Erin Eggleston ${ }^{2}$, \\ Ferran Garcia-Piche ${ }^{3}$ and Daniel H Buckley ${ }^{1}$ \\ ${ }^{1}$ Department of Crop and Soil Sciences, School of Integrative Plant Sciences, Cornell University, Ithaca, NY, \\ USA; ${ }^{2}$ Department of Microbiology, Cornell University, Ithaca, NY, USA and ${ }^{3}$ School of Life Sciences, Arizona \\ State University, Tempe, AZ, USA
}

\begin{abstract}
Biological soil crusts (BSCs) are key components of ecosystem productivity in arid lands and they cover a substantial fraction of the terrestrial surface. In particular, BSC $\mathrm{N}_{2}$-fixation contributes significantly to the nitrogen $(\mathrm{N})$ budget of arid land ecosystems. In mature crusts, $\mathrm{N}_{2}$-fixation is largely attributed to heterocystous cyanobacteria; however, early successional crusts possess few $\mathrm{N}_{2}$-fixing cyanobacteria and this suggests that microorganisms other than cyanobacteria mediate $\mathbf{N}_{2}$-fixation during the critical early stages of BSC development. DNA stable isotope probing with ${ }^{15} \mathrm{~N}_{2}$ revealed that Clostridiaceae and Proteobacteria are the most common microorganisms that assimilate ${ }^{15} \mathrm{~N}_{2}$ in early successional crusts. The Clostridiaceae identified are divergent from previously characterized isolates, though $\mathrm{N}_{2}$-fixation has previously been observed in this family. The Proteobacteria identified share $>98.5 \%$ small subunit rRNA gene sequence identity with isolates from genera known to possess diazotrophs (for example, Pseudomonas, Klebsiella, Shigella and Ideonella). The low abundance of these heterotrophic diazotrophs in BSCs may explain why they have not been characterized previously. Diazotrophs have a critical role in BSC formation and characterization of these organisms represents a crucial step towards understanding how anthropogenic change will affect the formation and ecological function of BSCs in arid ecosystems.
\end{abstract}

The ISME Journal (2016) 10, 287-298; doi:10.1038/ismej.2015.106; published online 26 June 2015

\section{Introduction}

Biological soil crusts (BSCs) are specialized microbial communities that form at the soil surface in arid environments and they fill a variety of important ecological functions. BSCs occupy plant interspaces and cover a wide, global geographic range (Garcia-Pichel et al., 2003). For example, $80 \%$ of the ground cover in some regions of the Colorado Plateau are covered by BSCs (Karnieli et al., 2003). The global biomass of BSC cyanobacteria alone is estimated at $54 \times 10^{12} \mathrm{~g} \mathrm{C}$ (GarciaPichel et al., 2003). BSC nitrogen fixation $\left(\mathrm{N}_{2}\right.$-fixation) is responsible for significant input of nitrogen $(\mathrm{N})$ to arid environments (Evans and Belnap, 1999; Belnap, 2001). Interestingly, much of this fixed $\mathrm{N}$ is exported from the crusts in dissolved form through percolation or runoff and little is lost to volatilization (Johnson et al., 2007). The presence of

Correspondence: DH Buckley, Department of Crop and Soil Sciences, School of Integrative Plant Sciences, Cornell University, 705 Bradfield Hall, Ithaca, NY 14853, USA.

E-mail: dhb28@cornell.edu

Received 4 December 2014; revised 19 May 2015; accepted 25 May 2015; published online 26 June 2015
BSCs is positively correlated with vascular plant survival due in part to $\mathrm{N}$ inputs from BSC (for review of BSC-vascular plant interactions, see Belnap et al., 2003). These microbial ecosystems are not immune to climate change and changes in precipitation and temperature could alter BSC microbial community structure/membership and possibly BSC diazotroph diversity and $\mathrm{N}_{2}$-fixation (Garcia-Pichel et al., 2013).

BSCs are highly susceptible to natural and anthropogenic disturbance (Garcia-Pichel et al., 2013). Succession in BSC communities is characterized by transition from early successional 'light' crusts to mature 'dark' crusts (Belnap, 2002; Yeager et al., 2004). Motile non-heterocystous cyanobacteria (for example, Microcoleus vaginatus or M. steenstrupii), which cannot fix $\mathrm{N}_{2}$, are pioneer colonizers of early successional crusts and are abundant in all types of BSCs (Yeager et al., 2004; Garcia-Pichel et al., 2013). Successional development of mature crust is accompanied by a change in color produced by secondary colonization with non-motile $\mathrm{N}_{2}$-fixing heterocystous cyanobacteria, which produce large amounts of sunscreen compounds that reduce soil albedo (Belnap, 2002; Yeager et al., 2004). These heterocystous cyanobacteria (for example, Scytonema, Spirirestis and Nostoc) increase in abundance during crust 
succession and are abundant in mature crusts (Yeager et al., 2007, 2012). Heterocystous cyanobacteria are numerically dominant in surveys of BSC nifH gene diversity (Yeager et al., 2007, 2004, 2012). For example, $89 \%$ of 693 nifH sequences derived from Colorado Plateau and New Mexico crusts were attributed to heterocystous cyanobacteria (Yeager et al., 2007). Other BSC nifH sequences are attributed to Alphaproteobacteria, Betaproteobacteria and Gammaproteobacteria, as well as a nifH clade (nifH cluster III) that includes diverse anaerobes, such as clostridia, sulfate-reducing bacteria and anoxygenic phototrophs (Steppe et al., 1996; Yeager et al., 2007).

Two lines of evidence suggest that nitrogen fixers other than phototrophs are important in early successional crusts. First, the contributions of early successional BSC to N2-fixation in arid ecosystems may have been systematically under-estimated. In mature crusts, heterocystous cyanobacteria are abundant at the crust surface and their presence corresponds with maximal rates of acetylene reduction (Johnson et al., 2005). However, in early successional crusts, rates of acetylene reduction are maximal below the crust surface and these communities possess few heterocystous cyanobacteria (Johnson et al., 2005). $\mathrm{N}_{2}$-fixation rates are typically determined by areal measurements made at the crust surface with the acetylene reduction assay. Rates determined in this manner vary significantly across samples and studies (Evans and Lange, 2001). The reasons for inter-site and inter-study variability are complex and likely include the spatial heterogeneity of BSCs (Evans and Lange, 2001). However, the acetylene reduction assay is also subject to methodological artifacts that can be affected by the physical and biological characteristics of samples (see Belnap, 2001 for a review). If BSC $\mathrm{N}_{2}$-fixation is estimated by integrating rates across a depth profile (which eliminates constraints from diffusional limitation), then total rates of $\mathrm{N}_{2}$-fixation do not differ significantly between early successional and mature BSCs (Johnson et al., 2005). This result suggests that diazotrophs other than heterocystous cyanobacteria are important contributors to $\mathrm{N}_{2}$-fixation in early successional BSC communities. Second, the bare soils that are colonized during the process of early crust formation are very low in $\mathrm{N}$ relative to mature crusts (Beraldi-Campesi et al., 2009), but the cyanobacteria that initially colonize new crusts (Microcoleus spp., Garcia-Pichel and Wojciechowski, 2009) are unable to fix nitrogen (Starkenburg et al., 2011; Rajeev et al., 2013) and hence the source of $\mathrm{N}_{2}$-fixation required for the establishment of early successional crusts remains poorly characterized.

To determine the agency of nitrogen fixation in early developmental crusts, we conducted ${ }^{15} \mathrm{~N}_{2}$ DNA stable isotope probing (DNA-SIP) experiments with early successional Colorado Plateau BSCs conspicuously devoid of significant surface populations of heterocystous cyanobacteria. DNA-SIP with ${ }^{15} \mathrm{~N}_{2}$ has not been previously attempted with BSCs. DNA-SIP provides an accounting of active diazotrophs on the basis of ${ }^{15} \mathrm{~N}_{2}$ assimilation into DNA, whereas nifH clone libraries merely account for microbes with the genomic potential for $\mathrm{N}_{2}$-fixation. Further, we investigate the distribution of these active diazotrophs in surveys of microbial diversity conducted on BSCs over a range of spatial scales and soil types (Garcia-Pichel et al., 2013; Steven et al., 2013).

\section{Materials and methods}

BSC sampling and incubation conditions

BSC samples were taken from the Green Butte site near Moab, Utah, USA as previously described (site 'CP3'; latitude N 3844'55.1", longitude W 10944'37.1"; Beraldi-Campesi et al., 2009). All samples were from early successional 'light' crusts as described by Johnson et al. (2005). Early successional BSC samples $\left(37.5 \mathrm{~cm}^{2}\right.$, average mass $\left.35 \mathrm{~g}\right)$ were incubated in sealed chambers under controlled atmosphere and in 16-h light/8-h dark for 4 days. Crusts were sampled and transported while dry and wetted at initiation of the experiment. Water was added to each sample to fully saturate the soil but avoid visible ponding. The samples were then placed in air-tight sealed incubation containers for the rest of the experiment, so that soil and atmosphere remained saturated through the incubation period. The water was amended with calcium bicarbonate to yield a final concentration of $3 \mathrm{~mm}$, so that autotrophy could proceed unimpeded. The control treatment received a headspace of air and the experimental treatment received a headspace containing ${ }^{15} \mathrm{~N}_{2}$ ( $>98 \%$ atom ${ }^{15} \mathrm{~N}_{2}$ ). ${ }^{15} \mathrm{~N}_{2}(100 \%)$ gas was purchased from Sigma-Aldrich (St Louis, MO, USA). We used a composition of $75 \%{ }^{15} \mathrm{~N}_{2}$ in helium for the initial incubation headspace. Four crust samples were treated and incubated (two control and two experimental). One control/experimental crust pair was collected at day 2 and the other at day 4. Acetylene reduction rates were measured daily. Acetylene reduction rates increased over the course of the experiment $(0.8,4.8,8.8$ and 14.5 $\mu$ moles $\mathrm{m}^{-2} \mathrm{~h}^{-1}$ ethylene for days $1-4$, respectively).

\section{DNA extraction}

DNA was extracted for DNA-SIP at 2 and 4 days. DNA was extracted from $1 \mathrm{~g}$ of BSCs. DNA from each sample was extracted using a MoBio (Carlsbad, CA, USA) UltraClean Mega Soil DNA Isolation Kit (following the manufacturer's protocol, but lysis was done as previously described (Strauss et al., 2011)), and then gel purified to select high molecular weight DNA $(>4 \mathrm{~kb})$ using a $1 \%$ low melt agarose gel and $\beta$-agarase I for digestion (according to the manufacturer's protocol, New England Biolabs, Ipswich, MA, USA, M0392S). Extracts were quantified using PicoGreen nucleic acid quantification dyes (Molecular Probes, Eugene, OR, USA). 
Formation of $\mathrm{CsCl}$ equilibrium density gradients $\mathrm{CsCl}$ gradient fractionation was used to separate the DNA into 36 gradient fractions on the basis of buoyant density. $\mathrm{CsCl}$ density gradients were formed in $4.7 \mathrm{ml}$ polyallomer centrifuge tubes filled with gradient buffer (15 mM Tris-HCl, pH 8; 15 mM EDTA; $15 \mathrm{mM} \mathrm{KCl}$ ), which contained $1.725 \mathrm{~g} \mathrm{ml}^{-1} \mathrm{CsCl}$. $\mathrm{CsCl}$ density was checked with a digital refractometer as described below. A total of 2.5-5.0 $\mu \mathrm{g}$ of DNA was added to each tube, and the tubes were mixed prior to centrifugation. Centrifugation was performed in a TLA-110 fixed angle rotor (Beckman Coulter, Indianapolis, IN, USA) at $20^{\circ} \mathrm{C}$ for $67 \mathrm{~h}$ at 55000 r.p.m. (Buckley et al., 2007). Centrifuged gradients were fractionated from bottom to top in 36 equal fractions of $100 \mu \mathrm{l}$, using a syringe pump as described previously (Buckley et al., 2007). The density of each fraction was determined using an AR200 refractometer (Reichert, Depew, NY, USA), modified to accommodate $5 \mu \mathrm{l}$ samples as described previously (Buckley et al., 2007). DNA in each fraction was desalted on a filter plate (PALL, Port Washington, NY, USA, AcroPrep Advance 96 Filter Plate, Product Number 8035), using four washes with $300 \mu \mathrm{l}$ TE per fraction. After each wash, the filter plate was centrifuged at $500 \mathrm{~g}$ for $10 \mathrm{~min}$, with a final spin of 20 min. Purified DNA from each fraction was resuspended in $50 \mu \mathrm{l}$ of TE buffer.

PCR, library normalization and DNA sequencing

To characterize the distribution of small subunit (SSU) rRNA genes across density gradients, SSU rRNA gene amplicons were generated from 20 gradient fractions per gradient for both unlabeled controls and ${ }^{15} \mathrm{~N}_{2}$ labeled samples. The 20 fractions analyzed are those expected to contain DNA (both labeled and unlabeled) having buoyant density in the range of 1.66-1.77 $\mathrm{g} \mathrm{ml}^{-1}$. Barcoded PCR of bacterial and archaeal SSU rRNA genes was carried out using primer set 515F/806R (Walters et al., 2011) (primers purchased from Integrated DNA Technologies, Coralville, IA, USA). The primer 806R contained an 8-bp barcode sequence, a 'TC' linker and a Roche $454 \mathrm{~B}$ sequencing adapter, while the primer $515 \mathrm{~F}$ contained the Roche 454A sequencing adapter. Each $25 \mu$ reaction contained $1 \times$ PCR Gold Buffer (Roche, Pleasanton, CA, USA), $2.5 \mathrm{~mm} \mathrm{MgCl}_{2}, 200 \mu \mathrm{m}$ of each of the four dNTPs (Promega, Madison, WI, USA), $0.5 \mathrm{mg} \mathrm{ml}^{-1}$ bovine serum albumin (New England Biolabs), $0.3 \mu \mathrm{M}$ of each primers, $1.25 \mathrm{U}$ of Amplitaq Gold (Roche), and $8 \mu \mathrm{l}$ of template. Each sample was amplified in triplicate. Thermal cycling occurred with an initial denaturation step of $5 \mathrm{~min}$ at $95^{\circ} \mathrm{C}$, followed by 40 cycles of amplification $\left(20 \mathrm{~s}\right.$ at $95^{\circ} \mathrm{C}$, $20 \mathrm{~s}$ at $53^{\circ} \mathrm{C}, 30 \mathrm{~s}$ at $72^{\circ} \mathrm{C}$ ), and a final extension step of $5 \mathrm{~min}$ at $72^{\circ} \mathrm{C}$. Triplicate amplicons were pooled and purified using Agencourt AMPure PCR purification beads (Beckman Coulter), following the manufacturer's protocol. Once purified, amplicons were quantified using PicoGreen nucleic acid quantification dyes (Molecular Probes) and pooled together in equimolar amounts. Samples were sent to the Environmental Genomics Core Facility at the University of South Carolina (now Selah Genomics) where they were run on a Roche FLX 454 pyrosequencing machine (FLX-Titanium platform, Roche).

\section{Data analysis}

Sequence quality control. Sequences were initially screened by maximum expected errors at a specific read length threshold (Edgar, 2013), and this has been shown to be as effective as denoising with respect to removing pyrosequencing errors. Specifically, reads were first truncated to 230 nucleotides (nt) (all reads shorter than $230 \mathrm{nt}$ were discarded) and any read that exceeded a maximum expected error threshold of 1.0 was removed. After truncation and maximum expected error trimming, 91\% of original reads remained. Forward primer and barcode were then removed from the high-quality, truncated reads. Remaining reads were taxonomically annotated using the 'UClust' taxonomic annotation framework in the QIIME software package (Caporaso et al., 2010; Edgar, 2010) with cluster seeds from Silva SSU rRNA database (Pruesse et al., 2007) 97\% sequence identity operational taxonomic units (OTUs) as reference (release SSU Ref 111). Reads annotated as 'Chloroplast', 'Eukaryota', 'Archaea', 'Unassigned' or 'mitochondria' were removed from the data set. Finally, reads were aligned to the Silva reference alignment provided by the Mothur software package (Schloss et al., 2009) using the Mothur NAST aligner (DeSantis et al., 2006). All reads that did not align to the expected amplicon region of the SSU rRNA gene were discarded. Quality control parameters removed 34716 of the 258763 raw reads. Raw sequences have been uploaded to MG-RAST (MG-RAST ID 4603397.3).

Sequence clustering. Sequences were distributed into OTUs using the UPARSE methodology (Edgar, 2013). Specifically, OTU centroids (that is, seeds) were identified using USEARCH on nonredundant reads sorted by count. The sequence identity threshold for establishing a new OTU centroid was $97 \%$. After initial OTU centroid selection, select SSU rRNA gene sequences from Yeager et al. (2007) were added to the centroid collection. Specifically, Colorado Plateau or Moab, Utah sequences from Yeager et al. (2007) sequences were added that included the SSU rRNA gene sequences for Calothrix MCC-3 A (accession DQ531700.1), Nostoc commune MCT-1 (accession DQ531903), Nostoc commune MFG-1 (accession DQ531699.1), Scytonema hyalinum DC-A (accession DQ531701.1), Scytonema hyalinum FGP-7 A (accession DQ531697.1) and Spirirestis rafaelensis LQ-10 (accession DQ531696.1). Original 
centroid sequences matching Yeager et al. (2007) sequences were subsequently removed from the centroid collection. With USEARCH/UPARSE, potential chimeras are identified during OTU centroid selection and are not allowed to become cluster centroids effectively removing chimeras from the read pool. All quality controlled reads were then mapped to cluster centroids at an identity threshold of $97 \%$ again using USEARCH. A total of $95.6 \%$ of quality controlled reads could be mapped to centroids. Unmapped reads do not count toward sample counts and were removed from downstream analyses. The USEARCH software version for cluster generation was 7.0.1090. The sequences form GarciaPichel et al. (2013) and Steven et al. (2013) were quality screened by alignment coordinates (described above) and included as input to USEARCH for OTU centroid selection and subsequent mapping to OTU centroids.

Phylogenetic analysis. Alignment of SSU rRNA genes was done with SSU-Align, which is based on Infernal (Nawrocki and Eddy, 2013; Nawrocki et al., 2009). Columns in the alignment that were not included in the SSU-Align covariance models or were aligned with poor confidence $(<95 \%$ of characters in a position had posterior probability alignment scores of at least $95 \%$ ) were masked for phylogenetic reconstruction. Additionally, the alignment was trimmed to coordinates such that all sequences in the alignment began and ended at the same positions. FastTree (Price et al., 2010) was used to build the tree.

Identifying OTUS that incorporated ${ }^{15} \mathrm{~N}$ into their DNA. DNA-SIP is a culture-independent approach toward defining identity-function connections in microbial communities (Radajewski and Murrell, 2001; Neufeld et al., 2007; Buckley, 2011). Microbes are identified on the basis of isotope assimilation into DNA. As the buoyant density of a macromolecule is dependent on many factors in addition to stable isotope incorporation (for example, G+Ccontent in nucleic acids; Youngblut and Buckley, 2014), labeled nucleic acids from one microbial population may have the same buoyant density as unlabeled nucleic acids from another. Therefore, it is imperative to compare results of isotopic labeling to results obtained with unlabeled controls where everything mimics the experimental conditions except that unlabeled substrates are used. By contrasting heavy gradient fractions from isotopically labeled samples relative to corresponding fractions from controls, the identities of microbes with labeled nucleic acids can be determined

We used an RNA-Seq differential expression statistical framework (Love et al., 2014) to find OTUs enriched in heavy fractions of labeled gradients relative to corresponding density fractions in control gradients (for review of RNA-Seq differential expression statistics applied to microbiome OTU count data, see McMurdie and Holmes (2014)). We use the term 'differential abundance' (coined by McMurdie and Holmes (2014)) to denote OTUs that have different proportion means across sample classes (in this case the only sample class is labeled:control). $\mathrm{CsCl}$ gradient fractions were categorized as 'heavy' or 'light'. The heavy category denotes fractions with density values $>1.725 \mathrm{~g} \mathrm{ml}^{-1}$. As we are only interested in enriched OTUs (labeled versus control), we used a one-sided Wald-test to test the statistical significance of regression coefficients (the null hypothesis is that the labeled:control fold enrichment for an OTU is less than a selected threshold). We independently filtered out sparse OTUs prior to $P$-value correction for multiple comparisons. The sparsity threshold was set to the value that maximized the number of $P$-values under a false discovery rate threshold. The specific sparsity threshold was 0.3 meaning that an OTU not found in at least $30 \%$ of heavy fractions (control and labeled gradients) in a given day were removed as statistically uninformative. $P$-values were corrected with the Benjamini-Hochberg method (Benjamini and Hochberg, 1995) and a false discovery rate of 0.10 was applied (this rate is the typical false discovery rate threshold adopted during RNASeq analysis). We selected a log2 fold change null threshold of 0.25 (or a labeled:control fold enrichment of 1.19). DESeq2 was used to calculate the moderated $\log 2$ fold change of labeled:control proportion means and corresponding s.es. for the Wald-test (above). Fold change moderation allows for reliable ranking such that high variance and likely statistically insignificant fold changes are appropriately shrunk and subsequently ranked lower than they would be as unmoderated values. Those OTUs that exhibit a statistically significant increase in proportion in heavy fractions from ${ }^{15} \mathrm{~N}_{2}$-labeled samples relative to corresponding controls have increased significantly in buoyant density in response to ${ }^{15} \mathrm{~N}_{2}$ treatment; a response that is expected for $\mathrm{N}_{2}$-fixing organisms.

We also assessed the consistency of enrichment between samples by including the interaction of day and label:control in a DESeq2 generalized linear model. The interpretation of the interaction coefficient is the change in OTU enrichment per unit time. $P$-values for the interaction coefficient were adjusted for all OTUs that passed the sparsity threshold in the label versus control comparison (above) and we used the default null model such that the coefficient equaled zero. Additionally, we assessed fold change between labeled and control gradient heavy fractions after pooling day-2 and day-4 data when treating the different time points as replicates. The same null model as the label versus control comparison (above) was used in this replicate analysis (log2 fold change in abundance between label and control is $\leqslant 0.25$ ). We included all OTUs that passed sparsity-based independent filtering at either day (above) for $P$-value adjustment in the replicate analysis. 

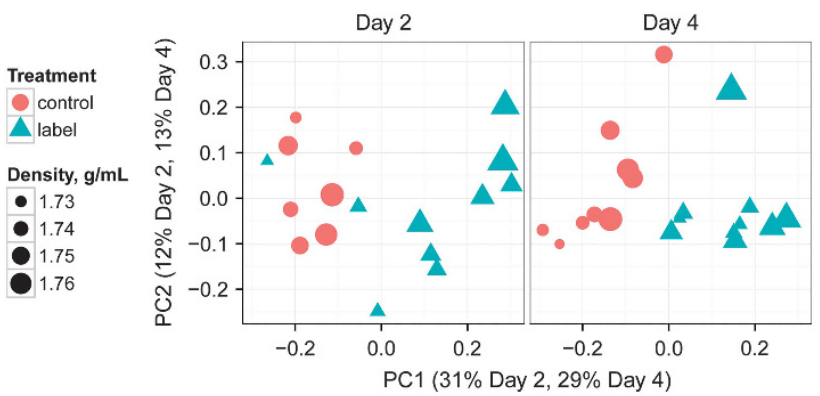

Figure 1 Ordination of heavy gradient fractions by Bray-Curtis distances on the basis of OTU content. Each point represents a gradient fraction OTU profile. Points closer together have more similar OTU content than those further apart.

Community and sequence analysis. BLAST searches were done with the 'blastn' program from BLAST+ toolkit (Camacho et al., 2009) version 2.2.29+. Default parameters were always employed and the BioPython (Cock et al., 2009) BLAST+ wrapper was used to invoke the blastn program. Pandas (McKinney, 2012) and dplyr (Wickham and Francois, 2014) were used to parse and manipulate BLAST output tables.

Principal coordinate ordinations depict the relationship between samples at each time point (days 2 and 4). Bray-Curtis distances were used as the sample distance metric for ordination. The Phyloseq (McMurdie and Holmes, 2014) wrapper for Vegan (Oksanen et al., 2013) (both R packages) was used to compute sample values along principal coordinate axes. GGplot2 (Wickham, 2009) was used to display sample points along the first and second principal axes. Adonis tests (Anderson, 2001) were done with default number of permutations (1000).

Rarefaction curves were created using bioinformatics modules in the PyCogent Python package (Knight et al., 2007). Parametric richness estimates were made with CatchAll using only the best model for total OTU estimates (Bunge, 2010).

All code to take raw sequencing data through the presented figures (including download and processing of literature environmental data sets) can be found at: https://github.com/chuckpr/NSIP data_analysis

\section{Results}

DNA buoyant density changes in response to ${ }^{15} \mathrm{~N}_{2}$ BSCs were wetted and incubated for 4 days in transparent chambers with headspace containing $\mathrm{N}_{2}$ either from air or from $100 \%$ atom-enriched ${ }^{15} \mathrm{~N}_{2}$. The chambers were illuminated with $16 \mathrm{~h}$ on/ $8 \mathrm{~h}$ off cycles at an intensity of $200 \mu \mathrm{mol}$ photons $\mathrm{m}^{-2} \mathrm{~s}^{-1}$, which is the equivalent of an overcast/rainy day. $\mathrm{N}_{2}$-fixation as measured by acetylene reduction increased from $4.8 \mu$ moles $\mathrm{m}^{-2}$ day $^{-1}$ on day 2 to $14.5 \mu_{\text {moles }}{ }^{-2}$ day $^{-1}$ on day 4. Amplicon sequences from ${ }^{15} \mathrm{~N}_{2}$-labeled samples and their corresponding unlabeled controls diverged specifically in heavy

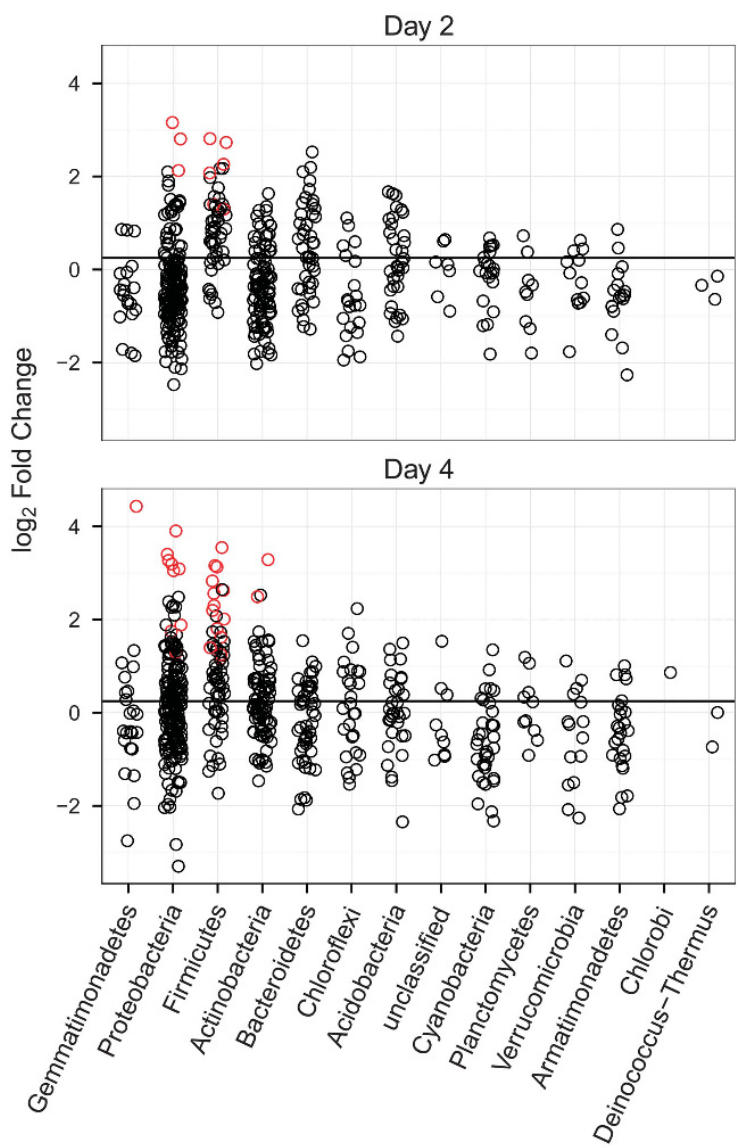

Phylum

Figure 2 Moderated log2 fold change of OTUs proportions for labeled versus control gradients (heavy fractions only, densities $>1.725 \mathrm{~g} \mathrm{ml}^{-1}$ ). All OTUs passing the sparsity threshold (see Materials and methods) at a specific incubation day are shown. Red color denotes a proportion fold change that has a corresponding adjusted $P$-value below a false discovery rate of $10 \%$ (ratio is significantly $>0.25$, black line).

gradient fractions (Figure 1 and Supplementary Figure S1) as assessed by Bray-Curtis dissimilarity (Bray and Curtis, 1957), and this result was significant (Adonis test (Anderson, 2001); P-value: $\left.0.001, r^{2}: 0.18\right)$.

OTUs responsive to ${ }^{15} \mathrm{~N}_{2}$ are primarily Proteobacteria and Clostridiaceae

OTUs that incorporated ${ }^{15} \mathrm{~N}$ into their DNA were detected by a differential change in their abundance within heavy gradient fractions of ${ }^{15} \mathrm{~N}_{2}$-labeled samples relative to corresponding controls. A total of 2127 and 2160 OTUs were detected in days 2 and 4, respectively, and these OTUs were interrogated for evidence of ${ }^{15} \mathrm{~N}_{2}$-labeling. Of these OTUs, only 499 and 563, respectively, passed a sparsity threshold applied to filter out OTUs with insufficient data for statistical analysis (see Love et al., 2014 for discussion of independent filtering). Of OTUs passing the sparsity criterion, 34 were enriched significantly in heavy fractions relative to control and 
a

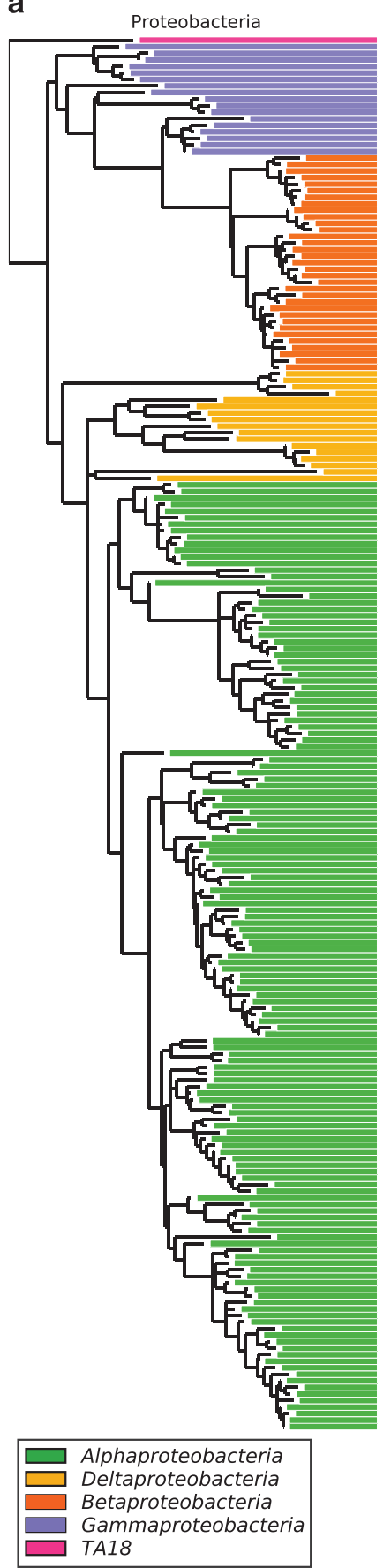

b

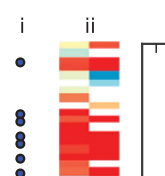

iii iv
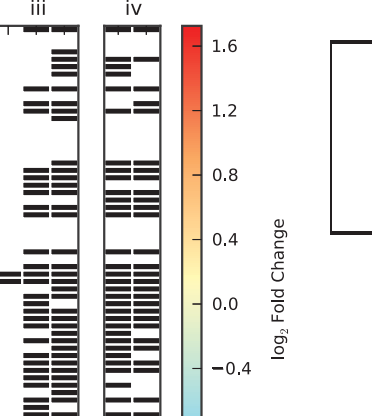

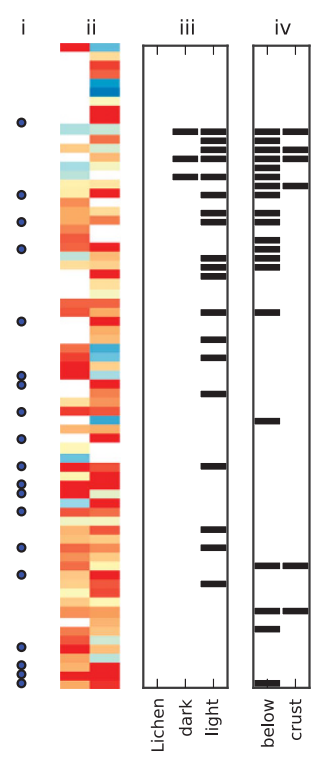

Figure 3 Phylogenetic trees of OTUs passing sparsity threshold for Proteobacteria (a) and Firmicutes (b). ${ }^{15} \mathrm{~N}$-responders are identified by dots present in column i. Log2 of OTU proportion fold change (labeled: control samples) for each OTU are presented as a heatmap in column ii, with results from days 2 and 4 on the left and right sides of the column, respectively. High fold change values indicate ${ }^{15} \mathrm{~N}$ incorporation. Presence/absence of OTUs (black indicates presence) in lichen, light or dark environmental samples (Garcia-Pichel et al., 2013) is shown in column iii. Presence/absence of OTUs (black indicates presence) in crust and below crust samples (Steven et al., 2013) is shown in column iv.

this result is specifically expected for OTUs that have ${ }^{15} \mathrm{~N}$-labeled DNA (that is, ${ }^{15} \mathrm{~N}_{2}$ 'responders'). Of these, 19 are annotated as Firmicutes, 12 as Proteobacteria, 2 as Actinobacteria and 1 as Gemmatimonadetes (Figures 2 and 3). If the responder OTUs are ranked by descending enrichment in heavy gradient fractions versus control, 8 of the top
10 responders (that is, those most enriched in the heavy fractions of labeled gradients) are either Firmicutes (3 OTUs) or Proteobacteria (5 OTUs) (Figure 4). Centroids (seed sequences) for strongly responding Proteobacteria OTUs all share high SSU rRNA gene sequence identity (>98.48\%, Supplementary Table S1) with isolates from genera 

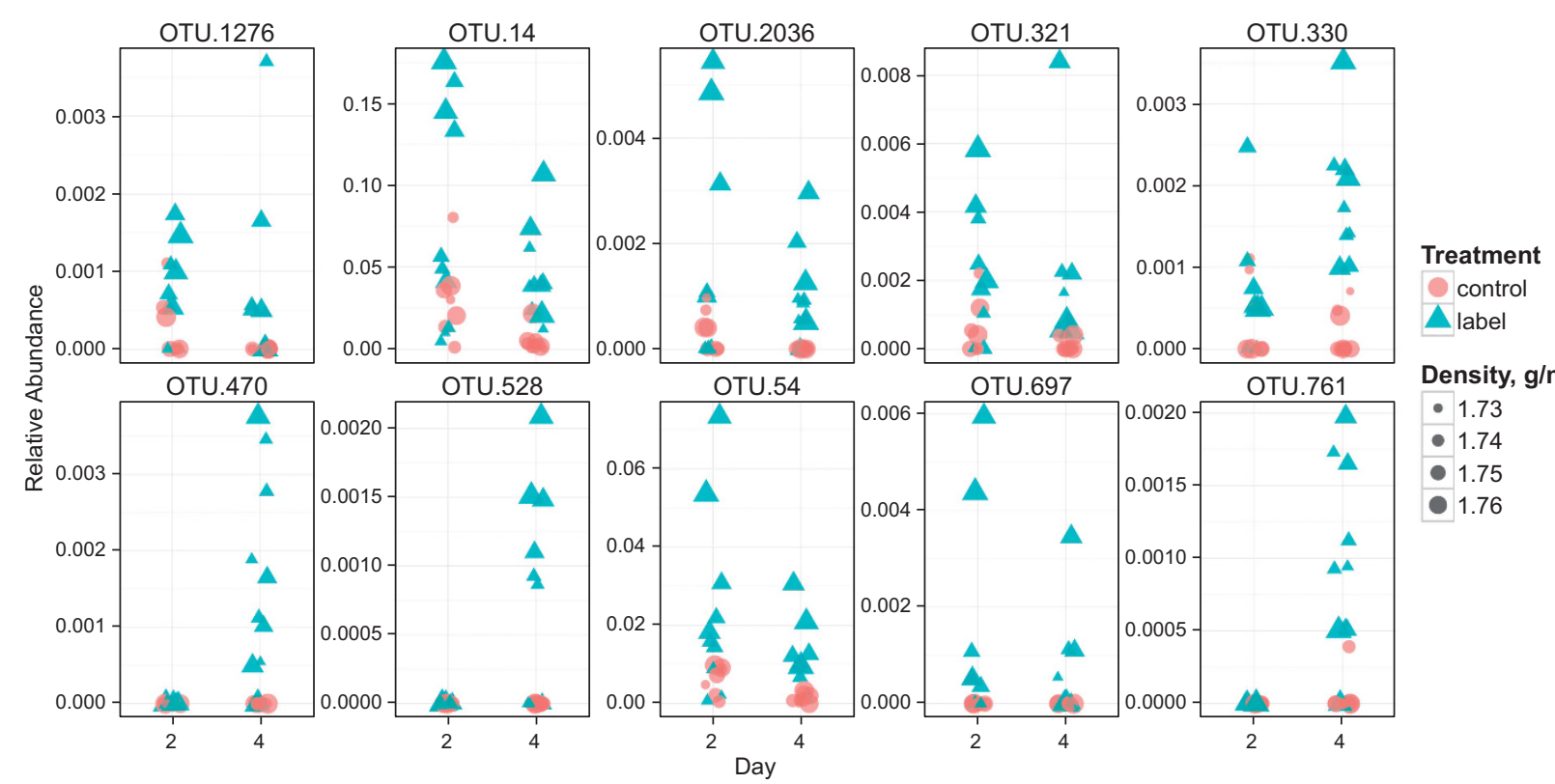

Density, $\mathrm{g} / \mathrm{mL}$

- 1.73

$\bullet 1.74$

1.75

- 1.76

Figure 4 Relative abundance values in heavy fractions (density $\geqslant 1.725 \mathrm{~g} \mathrm{ml}^{-1}$ ) for the top $10{ }^{15} \mathrm{~N}$ 'responders' (putative diazotrophs, see results for selection criteria of top 10) at each incubation day. Each point is a relative abundance value for the indicated OTU in a CsCl gradient fraction SSU rRNA gene collection. See Supplementary Table S1 for BLAST results against the LTP database (release 115). Point area is proportional to $\mathrm{CsCl}$ gradient fraction density, and color signifies control (red) or labeled (blue) treatment.

known to possess diazotrophs, including Pseudomonas, Klebsiella, Shigella and Ideonella. None of the Firmicutes OTU centroids in the top 10 responders share $>97 \%$ SSU rRNA gene sequence identity with sequences in the Living Tree Project (LTP) database of $16 \mathrm{~S}$ rRNA gene sequences from type strains (release 115; see Supplementary Table S1). OTUs that passed the sparsity threshold but were not classified as ${ }^{15} \mathrm{~N}$-responsive were subsequently tested with the null hypothesis that the OTU fold enrichment in labeled gradient heavy fractions versus control was above the selected threshold. Rejecting the second null indicates that an OTU did not incorporate ${ }^{15} \mathrm{~N}$ into biomass. There were 86 and 89 'non-responders' at days 2 and 4, respectively. The ${ }^{15} \mathrm{~N}$ labeling of OTUs that did not pass sparsity or could not be classified as either a responder or non-responder cannot be determined conclusively.

Although we did not take replicate samples within a time point, we can assess the consistency of each OTUs response across the two time points. OTU fold enrichment at days 2 and 4 was consistent (Supplementary Figure S2). There was a significant correlation between OTU fold enrichment at days 2 and $4\left(P=4.35 \mathrm{e}^{-8}\right)$. When the enrichment at day 2 is compared with day 4 via an interaction term (day $\times$ label/control, see Materials and methods), we find that only two OTUs have significantly different enrichment between the different times ('OTU.227' and 'OTU.4037', Supplementary Table S1). In addition, when day-2 and day-4 samples are treated as replicates (see Materials and methods) only five of the OTUs we identified as responders are not significantly enriched in labeled gradient heavy fractions versus control ('OTU.140', 'OTU.4037',
'OTU.227', 'OTU.137' and 'OTU.263', Supplementary Table S1). The labeling of these five OTUs should be interpreted with caution. In contrast, all other responders (Supplementary Table S1) showed consistent enrichment across time points based on the above analyses. Furthermore, confidence in detecting enrichment (as expressed by $P$-values between days 2 and 4 ) appears to be correlated with consistency in response across both days (Supplementary Figure S2).

${ }^{15} \mathrm{~N}$-responsive OTUs are found in low abundance in available environmental BSC SSU rRNA gene surveys In total, 13 of the $34{ }^{15} \mathrm{~N}$-responsive OTUs have been observed previously in SSU rRNA gene surveys of BSC communities (Figure 3 and Supplementary Figure S3). Eleven of the $19{ }^{15} \mathrm{~N}$-responsive Firmicutes OTUs are members of the Clostridiaceae. Three ${ }^{15} \mathrm{~N}$-responsive Clostridiaceae have been observed in previous BSC SSU rRNA gene surveys. Two ${ }^{15} \mathrm{~N}$-responsive Clostridiaceae were found in early successional crusts (Garcia-Pichel et al., 2013), and one ${ }^{15} \mathrm{~N}$-responsive Clostridiaceae OTU was found among the 'below crust' BSC SSU rRNA gene sequences described by Steven et al. (2013) (Figure 3). Five ${ }^{15} \mathrm{~N}$-responsive proteobacterial OTUs (Supplementary Table S1) were detected previously in BSC samples (Garcia-Pichel et al., 2013; Steven et al., 2013). The ${ }^{15} \mathrm{~N}$-responsive Gemmatimonadetes OTU was observed in four samples collected by Steven et al. (2013) and one ${ }^{15} \mathrm{~N}$-responsive Actinobacteria OTU was found in three samples collected by Steven et al. (2013). Gemmatimonadetes and Actinobacteria ${ }^{15} \mathrm{~N}$-responsive OTUs were not observed in samples collected by Garcia-Pichel et al. (2013). 
Comparison of SSU rRNA gene sequences from different BSC samples

We compared the SSU rRNA gene sequences determined in this DNA-SIP experiment with two previous surveys of SSU rRNA gene amplicons from BSC communities (Garcia-Pichel et al., 2013; Steven et al., 2013). There were 3079 OTUs (209 354 total sequences after quality control) in the DNA-SIP data, 3203 OTUs (129033 total sequences after quality control) in the study by Garcia-Pichel et al. (2013) and 2481 OTUs (129 358 total sequences after quality control) in the study by Steven et al. (2013) with a total of 4340 OTUs across all three data sets. Of the total 4340 OTU centroids established for this study, 445 have matches in the LTP (a collection of SSU rRNA gene sequences for all sequenced-type strains; Yarza et al., 2008) at or above a threshold of $97 \%$ sequence identity (LTP version 115). That is, 445 of the 4340 OTUs are closely related to known isolates. The DNA-SIP data shares $56 \%$ OTUs with the data by Steven et al. (2013) and $46 \%$ of OTUs with the data by Garcia-Pichel et al. (2013), while these latter two studies share $46 \%$ of their OTUs. This result suggests that low-frequency OTUs likely remain undersampled in all data sets.

Sequencing of DNA subjected to $\mathrm{CsCl}$ fractionation is expected to sample a different subset of diversity than that sampled by sequencing of unfractionated bulk DNA. For example, SIP enhances detection of OTUs that incorporate ${ }^{15} \mathrm{~N}$ into their DNA, and these OTUs will be overrepresented in the overall DNASIP sequence pool relative to their relative abundance in unfractionated bulk community samples. In addition, the DNA-SIP sequencing effort was directed at a relatively small number of light crust samples $(n=4)$, while previous sequencing efforts (Garcia-Pichel et al., 2013; Steven et al., 2013) were spread across hundreds of samples from both light and dark crusts. Hence, it is likely that the current study will be more likely to detect rare OTUs present in early successional light crust communities, particularly those that incorporate ${ }^{15} \mathrm{~N}$ into DNA. In all three BSC studies, most sequences were annotated as either cyanobacteria or Proteobacteria, though only in the DNA-SIP data did the sequences of Proteobacteria outnumber those of cyanobacteria. Proteobacteria represented $29.8 \%$ of sequence annotations in DNA-SIP data as opposed to $17.8 \%$ and $19.2 \%$ for the data by Garcia-Pichel et al. (2013) and Steven et al. (2013), respectively. In addition, sequences annotated as Firmicutes were more abundant in the DNA-SIP data $(19 \%)$ than in the data from Steven et al. (2013) and Garcia-Pichel et al. (2013) $(0.21 \%$ and $0.23 \%$, respectively; Supplementary Figure S4). Finally, as predetermined by sampling design, sequences annotated to 'Subsection IV' of cyanobacteria, which encompasses the heterocystous cyanobacteria in the Silva taxonomic nomenclature (Pruesse et al., 2007), comprised only $0.29 \%$ of cyanobacteria sequences in the DNA-SIP data while representing $15 \%$ and $23 \%$ of cyanobacteria sequences from the data by Steven et al. (2013) and Garcia-Pichel et al. (2013), respectively.

\section{Discussion}

BSC N-fixation has long been attributed to heterocystous cyanobacteria and the preponderance of cyanobacterial nifH genes observed in molecular surveys of BSCs have generally supported this hypothesis (Yeager et al., 2007, 2004, 2012). However, in this study ${ }^{15} \mathrm{~N}_{2}$-DNA-SIP reveals that non-cyanobacterial microorganisms fix $\mathrm{N}_{2}$ in early successional BSC samples. Proteobacteria and Clostridiaceae were most abundant among ${ }^{15} \mathrm{~N}_{2}$-responsive OTUs as revealed by a robust statistical framework for quantifying and evaluating differential OTU abundance in microbiome studies (Love et al., 2014; McMurdie and Holmes, 2014). Many of these OTUs (about 40\%) have been observed previously in BSC communities. Rarefaction curves of data from Steven et al. (2013) and Garcia-Pichel et al. (2013) are still sharply increasing especially for sub-crust samples (Supplementary Figure S5), suggesting that the communities remain undersampled. Parametric richness estimates of BSC diversity indicate that the sequencing efforts by Steven et al. (2013) and Garcia-Pichel et al. (2013) recovered on average $40.5 \%$ (s.d. $9.99 \%$ ) and $45.5 \%$ (s.d. $11.6 \%$ ) of predicted SSU rRNA gene OTUs from crust samples (inset, Supplementary Figure S5), respectively. Therefore, it would have been surprising if all of the ${ }^{15} \mathrm{~N}$-responsive OTUs had been observed in prior environmental surveys of BSCs. Nitrogenase nifH gene sequences related to both Proteobacteria and Clostridiaceae have been previously observed in BSC samples, though typically at relative abundance that is much lower than nifH gene sequences from heterocystous cyanobacteria.

We propose three mechanisms that could bias nifH clone libraries against heterotrophic diazotrophs. First, extreme polyploidy in cyanobacteria (up to $58 \times$ ploidy in stationary phase, Griese et al., 2011) can be expected to inflate the representation of cyanobacteria nifH gene sequences in community DNA relative to the frequency of ${ }^{15} \mathrm{~N}_{2}$-fixing heterocysts. Although cyanobacteria often have relatively large cells, ploidy per cell is probably greater than ploidy per unit volume. Second, heterocysts make up a small fraction of total cells along a trichome, though all cells in the trichome possess the nifH gene. As a result of polyploidy and heterocyst frequency in a cyanobacterial filament, the ratio of cyanobacterial nifH gene copies to heterotrophic nifH gene copies may be inflated as much as $10^{3}$ times relative to the corresponding ratio of ${ }^{15} \mathrm{~N}_{2}$ fixing cells (that is, the ratio of heterocyst number to the cell number of heterotrophic diazotrophs). Third, nifH PCR primers, which are highly degenerate, 
could be biased against heterotrophic diazotrophs. For example, the nifH PCR primers used in the second round of a widely used nested PCR protocol (Yeager et al., 2007, 2004, 2012) have fairly low coverage for Proteobacteria and Clostridiales (Gaby and Buckley, 2012). Primer 'nifH11' is biased against 'Cluster III' nifH gene sequences, which includes those of the Clostridiales (50\% in silico coverage of reference nifH sequences). In addition, primer 'nifH22' has low coverage of reference sequences from Proteobacteria, cyanobacteria and 'Cluster III' nifH gene sequences (16, 23 and $21 \%$ in silico coverage, respectively; Gaby and Buckley, 2012). Hence, it is reasonable to assume that heterotrophic diazotrophs may have been underestimated in previous analyses of early successional BSC communities. Our DNA-SIP results, which do not require PCR of functional genes, suggest that BSC N-fixation in early successional BSCs may include a large noncyanobacterial component. This is consistent with small-scale, spatially resolved functional measurements of N-fixation in BSCs (Johnson et al., 2005) that show a subsurface maximum that does not coincide spatially with maxima in chlorophyll $a$ (a proxy for phototrophic biomass) in early successional crusts and a surface maximum of $\mathrm{N}_{2}$-fixation in mature crust that coincides with the maximum in chlorophyll $a$.

We did not observe incorporation of ${ }^{15} \mathrm{~N}_{2}$ into the DNA of heterocystous cyanobacteria in the early successional BSC samples used in this study. It is possible that ${ }^{15} \mathrm{~N}_{2}$-fixation by heterocystous cyanobacteria could go undetected in DNA-SIP. One possible explanation for this result is that the early successional BSC samples used in this study possessed too few heterocystous cyanobacteria to statistically evaluate their ${ }^{15} \mathrm{~N}$-incorporation. Indeed, heterocystous cyanobacteria represented only $0.29 \%$ of sequences from the DNA-SIP data (see Results) as opposed to $15 \%$ and $23 \%$ of total sequences in the data by Steven et al. (2013) and Garcia-Pichel et al. (2013), respectively. OTUs that correspond to heterocystous cyanobacteria (as defined by Yeager et al., 2007) all fall below the sparsity threshold used in our analysis (see Materials and methods). Given the sparsity of heterocystous cyanobacteria sequences in the light crust DNA-SIP data, it is not possible to conclusively determine whether heterocystous cyanobacteria incorporated ${ }^{15} \mathrm{~N}$ during the incubation. Our results show that heterotrophic diazotrophs can contribute to ${ }^{15} \mathrm{~N}_{2}$-fixation in early successional BSCs but they do not exclude the potential for fixation by heterocystous cyanobacteria. Indeed, heterocystous cyanobacteria if present, active and limited for nitrogen would be expected to form heterocysts and fix ${ }^{15} \mathrm{~N}_{2}$. It is likely that scarcity limits their contribution to ${ }^{15} \mathrm{~N}_{2}$-fixation in early successional crusts. Heterocystous cyanobacteria form sessile colonies and they require stabilization of the crust environment before they can successfully colonize soil; and this stabilization is performed by other pioneering members of the crust community (Castenholz and GarciaPichel, 2002). ${ }^{15} \mathrm{~N}_{2}$-DNA-SIP would also fail to identify ${ }^{15} \mathrm{~N}_{2}$-fixing bacteria if ${ }^{15} \mathrm{~N}_{2}$-fixation were uncoupled from DNA replication over the time frame of the experiment (that is, 4 days), that is ${ }^{15} \mathrm{~N}_{2}$-DNA-SIP will not detect bacteria that fix ${ }^{15} \mathrm{~N}_{2}$ but do not incorporate the ${ }^{15} \mathrm{~N}$-label into DNA. Therefore, the contribution of heterocystous cyanobacteria (or any other microbe) to ${ }^{15} \mathrm{~N}_{2}$ would be underestimated if their cell division is uncoupled from ${ }^{15} \mathrm{~N}_{2}$-fixation at time frames of up to 4 days. We should also note that ${ }^{15} \mathrm{~N}$ can be incorporated into biomass from trophic interactions, although in this case the ${ }^{15} \mathrm{~N}$ labeling would likely be weaker than that for a $\mathrm{N}_{2}$-fixer as a result of label dilution.

The OTUs with significant evidence of ${ }^{15} \mathrm{~N}$-incorporation during the incubation were predominantly Proteobacteria and Firmicutes. The Proteobacteria OTUs with the strongest signal of ${ }^{15} \mathrm{~N}$-incorporation all shared high sequence identity $(\geqslant 98.48 \%$ ) with SSU rRNA gene sequences from genera known to contain diazotrophs (Supplementary Table S1). In contrast the Firmicutes that displayed signal for ${ }^{15} \mathrm{~N}$-incorporation (predominantly Clostridiaceae) were not closely related to any known cultivars (Supplementary Table S1). Hence, we have little knowledge of the ecology of these organisms. Assessing the physiological characteristics of these diazotrophic Clostridiaceae may be useful for predicting how environmental change will affect the development and stability of BSC. Prior intense cultivation efforts from these crusts in separate studies did not yield any members of the Clostridiaceae (Gundlapally and Garcia-Pichel, 2006). Although under sampled in environmental data sets, ${ }^{15} \mathrm{~N}$-responsive OTUs were indeed more abundant in sub-crust or early successional BSC samples relative to crust surface or mature crust samples (Figure 3 and Supplementary Figure S3). Although members of Clostridiaceae have been found in low abundance in molecular surveys of BSC, most surveys are carried out on desiccated crust samples, where thick-walled spores would predominate relative to vegetative cells, thus increasing the likelihood for their under-representation in DNA surveys. It should also be noted that crusts were incubated in an atmosphere of $\mathrm{He}$ and $\mathrm{N}_{2}$ rather than $\mathrm{O}_{2}$ and $\mathrm{N}_{2}$. Although cyanobacteria in the presence of light rapidly produce oxygen super saturation in BSCs relative to air (Garcia-Pichel and Belnap, 1996), and whereas heterotrophic $\mathrm{N}_{2}$-fixation by many microorganisms is inhibited in the presence of atmospheric levels of $\mathrm{O}_{2}$, it remains possible that the conditions present in microcosm are not representative of field conditions and may have favored $\mathrm{N}_{2}$-fixation by crust organisms that are less active in situ. Further experiments will need to be performed to verify that these heterotrophic diazotrophs are contributing to the N-budgets of early successional crusts in the field. 
Our results generate more refined hypotheses pertaining to the contribution of diazotrophs during the development of BSC communities. Specifically, ${ }^{15} \mathrm{~N}_{2}$-fixation in BSCs may not be tied solely to the climax of heterocystous cyanobacteria in mature crusts. Rather, ${ }^{15} \mathrm{~N}_{2}$-fixation may occur throughout crust development with the transition between early successional and mature crusts marked by a transition between heterotrophic and phototrophic ${ }^{15} \mathrm{~N}_{2}$-fixation in the crust community. Therefore, subbiocrust soil may contribute significantly to the arid ecosystem N-budget and may be of considerable importance in the early phases of BSC establishment. We propose that interactions between fast-growing heterotrophic diazotrophs such as members of the Clostridiaceae and filamentous (non-heterocystous) cyanobacteria are important in the early establishment of BSC communities. During progressive desiccation, cyanobacteria, such as $M$. vaginatus, accumulate compatible solutes, such as trehalose and sucrose (Rajeev et al., 2013). Upon wetting, microorganisms rapidly excrete compatible solutes to prevent cell lysis due to osmotic shock (Poolman and Glaasker, 1998). Among them are dihexoses (such as sucrose and trehalose), which are observed in natural crusts upon wetting and then are rapidly depleted in the soil solution (Northen, 2014). Many Clostridiaceae have a saccharolytic metabolism with the potential for rapid growth rates on substrates such as trehalose and/or sucrose (Wiegel et al., 2006). Wetting of crust may allow for rapid germination and growth of these organisms as the time required for germination of clostridial spores can be $<30 \mathrm{~min}$ (Stringer et al., 2005). Indeed, intense blooms of clostridia have been detected in crusts within tens of hours of wetting (Karaoz et al., 2014). $\mathrm{N}_{2}$-fixing clostridia are common in soils (Wiegel et al., 2006) and it is notable that Clostridium pasteurianum, isolated from soil, was the first $\mathrm{N}_{2}$-fixing bacterium ever described (Winogradsky, 1895). C. pasteurianum, though an anaerobe, grows readily in the presence of oxygen when co-cultured with aerobic organisms that reduce oxygen tension (Chester, 1903). We propose that, during a typical precipitation event, water saturation and heterotrophic activity rapidly render the interior of the crusts anoxic (Garcia-Pichel and Belnap, 1996), presenting optimal conditions for growth of anaerobic, dihexose-fermenting, $\mathrm{N}_{2}$-fixing clostridia. Clostridial organic nitrogen would then become available to other members of the community, including the primary producers, when carbon limitation induces sporulation and mother cell lysis. Mother cell lysis, the last step in sporulation, releases rich sources of $\mathrm{P}$ and $\mathrm{N}$ into the environment in the form of nucleotides and peptides (Hoch et al., 2002).

\section{Conclusion}

The abundance of ${ }^{15} \mathrm{~N}$-responsive OTUs from Clostrideaceae and Proteobacteria found in this study, the nifH gene sequences of Clostrideaceae and Proteobacteria observed previously in BSCs (Steppe et al., 1996) and the evidence for subsurface $\mathrm{N}_{2}$-fixation in early successional BSCs (Johnson et al., 2005), taken together, suggest that heterotrophic diazotrophs may be important contributors to $\mathrm{N}_{2}$-fixation in the subsurface of early successional BSCs. Heterocystous cyanobacteria are also key contributors to the BSC N-budget, however, and it is clear that heterocystous cyanobacteria increase in abundance with BSC's age (Yeager et al., 2004). It is less clear if the transition to mature crust is marked mainly by a change in the abundance and activity of heterocystous cyanobacteria or rather represents a succession within the diazotroph community from early crusts where ${ }^{15} \mathrm{~N}_{2}$-fixation is dominated by Clostridiaceae and Proteobacteria to mature crusts where it is dominated by heterocystous cyanobacteria. Predicting the ecological response of BSCs to climate change, altered precipitation regimes and physical disturbance requires an understanding of crust establishment, stability and succession. Diazotrophs are critical contributors to all of these phenomena and their activities make critical contributions to the N-budget of arid ecosystems worldwide.

\section{Conflict of Interest}

The authors declare no conflict of interest.

\section{Acknowledgements}

We thank T Whitman, CHD Williamson, AN Campbell and EK Hall for helpful comments in the preparation of this manuscript. This material is based upon work supported by the Department of Energy Office of Science, Office of Biological and Environmental Research Genomic Science Program under Award Numbers DE-SC0004486 and DE-SC0010558. This project was also supported by Agriculture and Food Research Initiative Competitive Grant no. 2007-35107-18299 from the USDA National Institute of Food and Agriculture. This report was prepared as an account of work sponsored by an agency of the United States Government. Neither the United States Government nor any agency thereof, nor any of their employees, makes any warranty, express or implied, or assumes any legal liability or responsibility for the accuracy, completeness or usefulness of any information, apparatus, product or process disclosed or represents that its use would not infringe privately owned rights. Reference herein to any specific commercial product, process or service by trade name, trademark, manufacturer or otherwise does not necessarily constitute or imply its endorsement, recommendation or favoring by the United States Government or any agency thereof. The views and opinions of authors expressed herein do not necessarily state or reflect those of the United States Government or any agency thereof.

\section{References}

Anderson M. (2001). A new method for non-parametric multivariate analysis of variance. Austral Ecol 26: 32-46. 
Belnap J. (2001). Factors influencing nitrogen fixation and nitrogen release in biological soil crusts. In: Belnap J, Lange O (eds). Biological Soil Crusts: Structure, Function, and Management. Ecological Studies, vol. 150. Springer: Berlin, Heidelberg, Germany, pp 241-261.

Belnap J. (2002). Nitrogen fixation in biological soil crusts from southeast Utah USA. Biol Fertil Soils 35: 128-135.

Belnap J, Prasse R, Harper K. (2003). Influence of biological soil crusts on soil environments and vascular plants. In: Belnap J, Lange O (eds). Biological Soil Crusts: Structure, Function, and Management. Ecological Studies, vol. 150. Springer: Berlin, Heidelberg, Germany, pp 281-300.

Benjamini Y, Hochberg Y. (1995). Controlling the false discovery rate: a practical and powerful approach to multiple testing. J R Stat Soc Ser B Stat Methodol 57: 289-300.

Beraldi-Campesi H, Hartnett H, Anbar A, Gordon G, Garcia-Pichel F. (2009). Effect of biological soil crusts on soil elemental concentrations: implications for biogeochemistry and as traceable biosignatures of ancient life on land. Geobiology 7: 348-359.

Bray J, Curtis J. (1957). An ordination of the upland forest communities of southern Wisconsin. Ecol Monogr 27: 325.

Buckley D. (2011). Stable isotope probing techniques using ${ }^{15} \mathrm{~N}$. In: Murrell J, Whiteley A (eds). Stable Isotope Probing and Related Technologies. American Society of Microbiology Press: Washington, DC, USA, pp 129-147.

Buckley D, Huangyutitham V, Hsu S, Nelson T. (2007). Stable isotope probing with $15 \mathrm{~N} 2$ reveals novel noncultivated diazotrophs in soil. Appl Environ Microbiol 73: 3196-3204.

Bunge J. (2010). Estimating the number of species with Catchall. In: Altman R, Dunker L, Murray H, Klein T (eds). Biocomputing 2011. World Scientific: Hackensack, NJ, USA, pp 121-130.

Camacho C, Coulouris G, Avagyan V, Ma N, Papadopoulos J, Bealer $\mathrm{K}$ et al. (2009). BLAST+: architecture and applications. BMC Bioinformatics 10: 421.

Caporaso J, Kuczynski J, Stombaugh J, Bittinger K, Bushman F, Costello E et al. (2010). QIIME allows analysis of high-throughput community sequencing data. Nat Methods 7: 335-336.

Castenholz RW, Garcia-Pichel F. (2002). Cyanobacteria responses to UV-radiation. In: Whitton B, Potts M (eds). The Ecology of Cyanobacteria. Springer: Dordrecht, The Netherlands, pp 591-611.

Chester F. (1903). Oligonitrophilic bacteria of the soil. Science 18: 370-371.

Cock P, Antao T, Chang J, Chapman B, Cox C, Dalke A et al. (2009). Biopython: freely available Python tools for computational molecular biology and bioinformatics. Bioinformatics 25: 1422-1423.

DeSantis TJ, Hugenholtz P, Keller K, Brodie E, Larsen N, Piceno $\mathrm{Y}$ et al. (2006). NAST: a multiple sequence alignment server for comparative analysis of $16 \mathrm{~S}$ rRNA genes. Nucleic Acids Res 34: W394-W399.

Edgar R. (2010). Search and clustering orders of magnitude faster than BLAST. Bioinformatics 26: 2460-2461.

Edgar R. (2013). UPARSE: highly accurate OTU sequences from microbial amplicon reads. Nat Methods 10: 996-998.

Evans R, Belnap J. (1999). Long-term consequences of disturbance on nitrogen dynamics in an arid ecosystem. Ecology 80: 150-160.
Evans R, Lange O. (2001). Biological soil crusts and ecosystem nitrogen and carbon dynamics. In: Belnap J, Lange O (eds). Biological Soil Crusts: Structure, Function, and Management. Ecological Studies, vol. 150. Springer: Berlin, Heidelberg, Germany, pp 263-279.

Gaby J, Buckley D. (2012). A comprehensive evaluation of PCR primers to amplify the nifH Gene of nitrogenase. PLoS One 7: e42149.

Garcia-Pichel F, Belnap J. (1996). Microenvironments and microscale productivity of cyanobacterial desert crusts. J Phycol 32: 774-782.

Garcia-Pichel F, Belnap J, Neuer S, Schanz F. (2003). Estimates of global cyanobacterial biomass and its distribution. Algol Stud 109: 213-227.

Garcia-Pichel F, Johnson S, Youngkin D, Belnap J. (2003). Small-scale vertical distribution of bacterial biomass and diversity in biological soil crusts from arid lands in the Colorado Plateau. Microb Ecol 46: 312-321.

Garcia-Pichel F, Loza V, Marusenko Y, Mateo P, Potrafka R. (2013). Temperature drives the continental-scale distribution of key microbes in topsoil communities. Science 340: 1574-1577.

Garcia-Pichel F, Wojciechowski MF. (2009). The evolution of a capacity to build supra-cellular ropes enabled filamentous cyanobacteria to colonize highly erodible substrates. PLoS One 4: e7801.

Griese M, Lange C, Soppa J. (2011). Ploidy in cyanobacteria. FEMS Microbiol Lett 323: 124-131.

Gundlapally SR, Garcia-Pichel F. (2006). The community and phylogenetic diversity of biological soil crusts in the colorado plateau studied by molecular fingerprinting and intensive cultivation. Microb Ecol 52: 345-357.

Hoch J, Sonenshein A, Losick R. (2002). Bacillus subtilis: From cells to genes and from genes to cells. In: Sonenshein A, Hoch J, Losick R (eds). Bacillus subtilis and its Closest Relatives. American Society of Microbiology: Washington, DC, USA.

Johnson S, Budinoff C, Belnap J, Garcia-Pichel F. (2005). Relevance of ammonium oxidation within biological soil crust communities. Environ Microbiol 7: 1-12.

Johnson SL, Neuer S, Garcia-Pichel F. (2007). Export of nitrogenous compounds due to incomplete cycling within biological soil crusts of arid lands. Environ Microbiol 9: 680-689.

Karaoz U, Estelle C, Nunes Da Rocha U, Northen T, Garcia-Pichel F, Brodie E. (2014). In preparation.

Karnieli A, Kokaly R, West N, Clark R. (2003). Remote sensing of biological soil crusts. In: Belnap J, Lange O (eds). Biological Soil Crusts: Structure, Function, and Management. Ecological studies, vol. 150. Springer: Berlin Heidelberg, Germany, pp 431-455.

Knight R, Maxwell P, Birmingham A, Carnes J, Caporaso J, Easton B et al. (2007). PyCogent: a toolkit for making sense from sequence. Genome Biol 8: R171.

Love MI, Huber W, Anders S. (2014). Moderated estimation of fold change and dispersion for RNA-Seq data with DESeq2. Genome Biol 15: 550.

McKinney W. (2012). Pandas: python data analysis library. http://pandas.pydata.org/.

McMurdie P, Holmes S. (2014). Waste not, want not: why rarefying microbiome data is inadmissible. PLoS Comput Biol 10: e1003531.

Nawrocki E, Eddy S. (2013). Infernal 1.1: 100-fold faster RNA homology searches. Bioinformatics 29: 2933-2935. 
Nawrocki E, Kolbe D, Eddy S. (2009). Infernal 1.0 inference of RNA alignments. Bioinformatics 25: 1335-1337.

Neufeld J, Vohra J, Dumont M, Lueders T, Manefield M, Friedrich $\mathrm{M}$ et al. (2007). DNA stable-isotope probing. Nat Protoc 2: 860-866.

Northen T. (2014). Personal communication.

Oksanen J, Blanchet F, Kindt R, Legendre P, Minchia NP, O'Hara $\mathrm{R}$ et al. (2013). Vegan: community ecology package. http://CRAN.R-project.org/package=vegan.

Poolman B, Glaasker E. (1998). Regulation of compatible solute accumulation in bacteria. Mol Microbiol 29: 397-407.

Price M, Dehal P, Arkin A. (2010). FastTree 2-approximately maximum-likelihood trees for large alignments. PLoS One 5: e9490.

Pruesse E, Quast C, Knittel K, Fuchs B, Ludwig W, Peplies J et al. (2007). SILVA: a comprehensive online resource for quality checked and aligned ribosomal RNA sequence data compatible with ARB. Nucleic Acids Res 35: 7188-7196.

Radajewski S, Murrell J. (2002). Stable isotope probing for detection of methanotrphs after enrichment with ${ }^{13} \mathrm{CH}_{4}$. In de Muro MA, Rapley R (eds), Gene Probes. Humana Press: New York, NY, USA, pp 149-157.

Rajeev L, Rocha UN, da, Klitgord N, Luning EG, Fortney J, Axen SD et al. (2013). Dynamic cyanobacterial response to hydration and dehydration in a desert biological soil crust. ISME J 7: 2178-2191.

Schloss P, Westcott S, Ryabin T, Hall J, Hartmann M, Hollister E et al. (2009). Introducing Mothur: opensource, platform-independent, community-supported software for describing and comparing microbial communities. Appl Environ Microbiol 75: 7537-7541.

Starkenburg SR, Reitenga KG, Freitas T, Johnson S, Chain PSG, Garcia-Pichel F et al. (2011). Genome of the cyanobacterium Microcoleus vaginatus FGP-2 a photosynthetic ecosystem engineer of arid land soil biocrusts worldwide. J Bacteriol 193: 4569-4570.

Steppe T, Olson J, Paerl H, Litaker R, Belnap J. (1996). Consortial N2 fixation: a strategy for meeting nitrogen requirements of marine and terrestrial cyanobacterial mats. FEMS Microbiol Ecol 21: 149-156.

Steven B, Gallegos-Graves L, Belnap J, Kuske C. (2013). Dryland soil microbial communities display spatial biogeographic patterns associated with soil depth and soil parent material. FEMS Microbiol Ecol 86: 101-113.

Strauss SL, Day TA, Garcia-Pichel F. (2011). Nitrogen cycling in desert biological soil crusts across biogeographic regions in the southwestern united states. Biogeochemistry 108: 171-182.

Stringer SC, Webb MD, George SM, Pin C, Peck MW. (2005). Heterogeneity of times required for germination and outgrowth from single spores of nonproteolytic Clostridium botulinum. Appl Environ Microbiol 71: 4998-5003.

Walters W, Caporaso J, Lauber C, Berg-Lyons D, Fierer N, Knight R. (2011). PrimerProspector: de novo design and taxonomic analysis of barcoded polymerase chain reaction primers. Bioinformatics 27: 1159-1161.

Wickham H. (2009). ggplot2: Elegant Graphics for Data Analysis. Springer: New York, NY, USA, http://had.co. nz/ggplot2/book.

Wickham H, Francois R. (2014). dplyr: a grammar of data manipulation. http://CRAN.R-project.org/package=dplyr.

Wiegel J, Tanner R, Rainey F. (2006). An introduction to the family Clostridiaceae. In: Rosenberg E, DeLong E, Lory S, Stackebrandt E, Thompson F (eds). The Prokaryotes. Springer: New York, NY, USA, pp 654-678.

Winogradsky S. (1895). Recherches sur l'assimilation de l'azote libre de l'atmosphère par les microbes. Arch Sci Biol 4: 297.

Yarza P, Richter M, Peplies J, Euzeby J, Amann R, Schleifer K et al. (2008). The All-Species Living Tree project: A $16 \mathrm{~S}$ rRNA-based phylogenetic tree of all sequenced type strains. Syst Appl Microbiol 31: 241-250.

Yeager C, Kornosky J, Housman D, Grote E, Belnap J, Kuske C. (2004). Diazotrophic community structure and function in two successional stages of biological soil crusts from the Colorado Plateau and Chihuahuan Desert. Appl Environ Microbiol 70: 973-983.

Yeager C, Kornosky J, Morgan R, Cain E, Garcia-Pichel F, Housman D et al. (2007). Three distinct clades of cultured heterocystous cyanobacteria constitute the dominant N2-fixing members of biological soil crusts of the Colorado Plateau USA. FEMS Microbiol Ecol 60: 85-97.

Yeager C, Kuske C, Carney T, Johnson S, Ticknor L, Belnap J. (2012). Response of biological soil crust diazotrophs to season altered summer precipitation, and year-round increased temperature in an arid grassland of the Colorado Plateau, USA. Front Microbiol 3: 358.

Youngblut ND, Buckley DH. (2014). Intra-genomic variation in $\mathrm{G}+\mathrm{C}$ content and its implications for dNA stable isotope probing. Environ Microbiol Rep 6: $767-775$.

Supplementary Information accompanies this paper on The ISME Journal website (http://www.nature.com/ismej) 